\title{
Effects of mulberry leaf on experimental hyperlipidemia rats induced by high-fat diet
}

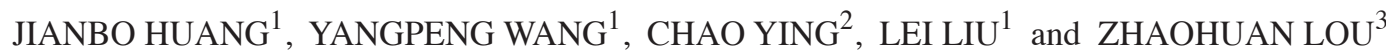 \\ ${ }^{1}$ Department of Basic Theory of Traditional Chinese Medicine, College of Basic Medical Science, \\ Zhejiang Chinese Medical University, Hangzhou, Zhejiang 310053; ${ }^{2}$ Institute of Materia Medica, \\ College of Pharmaceutical Science, Wenzhou Medical University, Wenzhou, Zhejiang 325035; \\ ${ }^{3}$ Institute of Materia Medica, College of Pharmaceutical Science, Zhejiang Chinese Medical University, \\ Hangzhou, Zhejiang 310053, P.R. China
}

Received October 2, 2017; Accepted March 9, 2018

DOI: $10.3892 /$ etm.2018.6254

\begin{abstract}
Hypercholesterolemia is a major risk factor for cardiovascular disease. Mulberry leaf (ML) is a Traditional Chinese Medicine used to treat hyperlipidemia in clinical settings. The aim of the present study was to identify the potential effect and possible target of ML in anti-hypercholesterolemia. Male Sprague-Dawley rats were fed with a high-fat diet and treated with ML for 5 weeks. Blood lipid levels, total cholesterol (TC) and total bile acid (TBA) in the liver and feces were measured to assess the effects of ML on hypercholesterolemia. Harris's hematoxylin staining and oil red $\mathrm{O}$ staining was applied to observe the pathological change and lipid accumulation in the liver. Immunohistochemical assay was performed to observe the location of expressions of scavenger receptor class B type I and low-density lipoprotein (LDL) receptor (-R), and western blotting was applied to determine the protein expression of ATP-binding cassette transporter G5/G8 (ABCG5/8), nuclear transcription factor peroxisome proliferator-activated receptor- $\alpha(\operatorname{PPAR} \alpha)$, farnesoid-X receptor (FXR) and cholesterol $7 \alpha$-hydroxylase 1 (CYP7A1). The results demonstrated that ML treatment reduced serum TC and LDL-cholesterol levels, and liver TC and TBA contents; increased serum HDL-C levels, and fecal TC and TBA contents; and alleviated hepatocyte lipid degeneration. In addition, ML treatment inhibited liver LDL-R, PPAR $\alpha$ and FXR protein expression, promoted protein expression of CYP7A1, and maintained the ratio of ABCG5/ABCG8. The findings of the present study provide a positive role of ML on cholesterol clearance via promoting cholesterol and
\end{abstract}

Correspondence to: Dr Zhaohuan Lou, Institute of Materia Medica, College of Pharmaceutical Science, Zhejiang Chinese Medical University, 548 Binwen Road, Binjiang, Hangzhou, Zhejiang 310053, P.R. China

E-mail: zhaohuanlou@zcmu.edu.cn

Key words: hyperlipidemias, reverse cholesterol transport, bile acid, cholesterol $7 \alpha$-hydroxylase 1
TBA execration via FXR- and CYP7A1-mediated pathways; $\mathrm{RCT}$ regulation may be a potential mechanism of $\mathrm{ML}$ on anti-hypercholesterolemia.

\section{Introduction}

It is widely known that hyperlipidemia, particularly hypercholesterolemia, is a notable risk factor for the development of cardiovascular disease (CVD), which is the main cause of mortality worldwide (1), and was recently identified as a causative factor for rotator cuff tear (2). At present, statins, such as lovastatin and atorvastatin, are the most commonly used lipid-lowering drugs, as they efficiently reduce plasma lipids; however, they also present a number of undesirable side effects, such as hepatotoxicity (3), rhabdomyolysis (4) and skeletal muscle injury (5), which have limited their usage. Therefore, it is necessary to identify and develop effective and natural agents that may be valuable in regulating lipid metabolism. In recent years, Traditional Chinese Medicine has attracted greater attention in metabolic syndrome treatments, and has become a common therapy for controlling symptoms in patients with hyperlipidemia (6).

Mulberry leaf (ML), the leaf of Morus alba L., is a Traditional Chinese Medicine that has been used in clinical settings in China for decades. Previous reports have demonstrated that ML extract and powder exhibit anti-type 2 diabetes (7-9), antioxidant (10,11), anti-inflammatory (12), anti-obesity $(13,14)$ and anti-atherosclerosis $(15)$ properties. A number of previous studies have demonstrated that $\mathrm{ML}$ powder was effective in reducing serum triglyceride (TG) and low-density lipoprotein (LDL)-cholesterol (C) in patients with mild hyperlipidemia $(16,17)$ and in hyperlipidemia rats (18). In addition, ML may significantly decrease plasma total cholesterol (TC) and increase high-density lipoprotein (HDL)-C (19,20). Valacchi et al (21) have previously reported that a combination of ML and mulberry fruit had a beneficial effect on the regulation of cholesterol transport via modulating the expression of scavenger receptor class $B$ type I (SR-BI) and ATP-binding cassette transporter A1 (ABCA1) in obese mice. As HDL-C is a key factor in reverse cholesterol transport (RCT) (22), which is a mechanism of 
cholesterol clearance in vivo (23), and SR-BI and ABCA1 are RCT-related proteins, it was hypothesized that ML may regulate the process of RCT to reduce serum cholesterol levels in patients with hyperlipidemia. The aim of the present study was to confirm the effects of ML on serum cholesterol reduction and RCT-related protein expression to identify the potential target.

\section{Materials and methods}

Reagents. ML powder was produced by Shaoxing Royal Tea Village Co., Ltd., (Shaoxing, China) via a process of hot air drying and ball milling technology, and the content of total flavonoids in the powder was $53 \%$ which was measured by ultraviolet-visible spectroscopy. ML powder was suspended in pure water according to the dosage with the final concentration as $0.09,0.06$ and $0.03 \mathrm{~g} / \mathrm{ml}$ prior to intragastric administration. Biochemical assay kits for TC (15110401), TG (15090101), HDL-C (15091401), LDL-C (15100703), aspartate aminotransferase (AST; 15092901), alanine aminotransferase (ALT; 15092401), were purchased from Medicalsystem Biotechnology Co., Ltd (Ningbo, China). ELISA kits for the determination of TG (cat. no. 15090101), TC (cat. no. 20151127) and total bile acid (TBA; 20151128) in the liver and feces were obtained from Nanjing Jiancheng Bioengineering Institute (Nanjing, China). High-fat diet (HFD) consisted of standard fodder (82\%), lard (10\%), cholesterol (1\%), cholate (1\%) and yolk powder $(5 \%)$ and was produced by Trophic Animal Feed High-tech Co., Ltd. (Nantong, China).

Animals and treatments. A total of 48 male Sprague-Dawley (SD) rats (age, 6-8 weeks; weight, 180-200 g) were purchased from the Animal Supply Center of Zhejiang Academy of Medical Science [Hangzhou, China; certificate no. SCXK (Zhe) 2014-0001]. Rats were housed in an environmentally controlled breeding room (temperature, $25 \pm 1^{\circ} \mathrm{C}$; humidity, $55 \pm 5 \%$; 12-h light/dark cycle) for one-week acclimatization prior to experiments. All rats were fed rodent laboratory chow with tap water ad libitum, and were fasted for $12 \mathrm{~h}$ prior to experiments with ad libitum access to water. All procedures were performed in strict accordance with the P.R. China Legislation on the Use and Care of Laboratory Animals (24) and with the Animal Management Rules of the Health Ministry of China (25). The present study was approved by the Ethics Committee of Zhejiang Chinese Medical University (Hangzhou, China).

$\mathrm{SD}$ rats were divided into six groups (n=8/group) including the normal control (normal), model control (model), atorvastatin (positive control) and three ML-treated $(0.9,0.6,0.3 \mathrm{~g} / \mathrm{kg}$ ) groups. Animals were given ad libitum access to HFD for 5 weeks, except for the normal group, which were administered the control diet. At the same time, normal and model groups were orally administered distilled water, whereas the atorvastatin and ML groups were administered with $6.0 \mathrm{mg} / \mathrm{kg}$ atorvastatin (China Meheco Group Co., Ltd., Beijing, China), or $0.9,0.6$ or $0.3 \mathrm{~g} / \mathrm{kg} \mathrm{ML}$ on a daily basis. At the end of experiment all rats were anesthetized and sacrificed. Livers were harvested for histopathology, immunohistochemical staining and western blot analysis.
Biochemical analysis and liver TC, TG and TBA assay. Following sacrifice, blood was collected from rat abdominal aortas and centrifuged at $206 \mathrm{x} \mathrm{g}$ for $15 \mathrm{~min}$ at $4^{\circ} \mathrm{C}$ to separate the serum. Serum TC, TG, HDL-C, LDL-C, ALT and AST levels were measured using a fully automatic blood biochemistry analyzer (TBA-40FR; Toshiba Medical Systems Corporation, Otawara, Japan) with commercial biochemical kits according to the manufacturer's protocol. Liver homogenate was prepared by grinding $0.5 \mathrm{~g}$ liver tissue in $4.5 \mathrm{ml}$ absolute ethyl alcohol followed by centrifugation at $825 \mathrm{x} \mathrm{g}$ for $10 \mathrm{~min}$ and the supernatant was separated. Levels of TC, TG and TBA in the liver were measured using ELISA kits and a PowerWave 340 microplate reader (BioTek Instruments, Inc., Winooski, VT, USA), according to the manufacturer's protocol.

Fecal TC and TBA levels determination. Prior to sacrifice, fecal samples were collected from each rat and dried in an oven at $\leq 80^{\circ} \mathrm{C}$. Subsequently, $0.3 \mathrm{~g}$ fecal powder was extracted with $3.4 \mathrm{ml}$ absolute ethyl alcohol using an ultrasonic apparatus (KQ-500DE; Kunshan Ultrasonic Instruments Co., Ltd., Kunshan, China) followed by centrifugation at $825 \mathrm{x} \mathrm{g}$ for $10 \mathrm{~min}$ at room temperature, and the supernatant was separated as the sample for determination. TC and TBA levels were measured using ELISA kits and the PowerWave 340 microplate reader according to the manufacturer's protocol.

Oil red O/hematoxylin staining. The Oil red $\mathrm{O} /$ hematoxylin staining procedure was performed at room temperature as follows: $5-\mu \mathrm{m}$ frozen liver sections were fixed in $4 \%$ paraformaldehyde solution for $10 \mathrm{~min}$, the sections were washed with PBS and stained with Oil red O (WSIG20100803; Sinopharm Chemical Reagent Co., Ltd., Shanghai, China) for $15 \mathrm{~min}$, followed by washing with PBS and staining with Harris's hematoxylin (20151216; Nanjing Jiancheng Bioengineering Institute) staining for $3 \mathrm{~min}$. Lipid droplets were observed under a light microscope (magnification, x200; B5-223IEP; Motic China Group Co., Ltd., Xiamen, China).

Liver histopathological analysis. Liver tissues were fixed in $10 \%$ neutral-buffered formalin for 1 week at $25-27^{\circ} \mathrm{C}$, dehydrated in a $70-100 \%$ gradient of ethyl alcohol, washed in xylene, embedded in paraffin and cut into $5-\mu \mathrm{m}$ sections. The liver sections were deparaffinized in xylene, rehydrated in a reverse-gradient series of ethyl alcohol, and stained with hematoxylin for $5 \mathrm{~min}$ and eosin for $5 \mathrm{~min}$ (H\&E; Merck KGaA, Darmstadt, Germany) at room temperature. Pathological changes were observed under a light microscope (magnification, x200), and analyzed with Motic Images Advanced 3.2 software (Motic China Group Co., Ltd.).

Immunohistochemistry analysis. The location of SR-BI and low-density lipoprotein LDL-receptor (LDL-R) in liver tissue was evaluated via immunohistochemistry. In brief, liver specimens fixed in $10 \%$ formalin at room temperature for 1 week, were embedded in paraffin wax, cut into $5-\mu \mathrm{m}$-thick sections, deparaffinized in xylene and rehydrated in a reverse-gradient series of ethyl alcohol. Following treatment with $3 \%$ hydrogen peroxide for $15 \mathrm{~min}$ at room 
temperature to block endogenous peroxidase activity, the sections were incubated with primary antibodies against SR-BI (1:100; ab52629; Abcam, Cambridge, UK) and LDL-R (1:100; sc-11824; Santa Cruz Biotechnology, Inc., Dallas, TX, USA) for $12 \mathrm{~h}$ at $4^{\circ} \mathrm{C}$ then washed with PBS. Biotinylated goat anti-polyvalent secondary antibodies [1:200; Mouse and Rabbit Specific HRP/DAB (ABC) Detection IHC kit; ab64264; Abcam] were added and incubated for $20 \mathrm{~min}$ at room temperature. The specimens were subsequently incubated with streptavidin peroxidase for $10 \mathrm{~min}$ at room temperature and DAB (1:50) was applied to visualize the labeling. The positive area was identified with brown staining under the B5-223IEP light microscope (magnification, $\mathrm{x} 400$ ).

Western blot analysis. Liver tissues $(\sim 100 \mathrm{mg})$ were homogenized with liquid nitrogen, lysed with radioimmunoprecipitation buffer (P0013B; Beyotime Institute of Biotechnology, Haimen, China) and protease/phosphatase inhibitors for $30 \mathrm{~min}$ on ice, and centrifuged at $18,246 \mathrm{x}$ g for $15 \mathrm{~min}$ at $4^{\circ} \mathrm{C}$. Total proteins were quantified using the BCA method with a protein quantitation kit (P0012; Beyotime Institute of Biotechnology). Protein samples $(50 \mu \mathrm{g} /$ lane $)$ were separated by $10 \%$ SDS-PAGE and transferred onto polyvinylidene fluoride membranes. The membranes were blocked with $5 \%$ skimmed milk in Tris-buffered saline containing $0.05 \%$ Tween-20 (TBST) for $120 \mathrm{~min}$ at room temperature. Following overnight incubation at $4^{\circ} \mathrm{C}$ with the following primary antibodies: Peroxisome proliferator-activated receptor- $\alpha$ (PPAR $\alpha ; 1: 1,000$; 15540-1-AP, ProteinTech Group, Inc., Chicago, IL, USA), SR-BI (1:1,000; ab52629, Abcam), LDL-R (1:1,000; sc-11824, Santa Cruz Biotechnology, Inc.), ATP-binding cassette transporter (ABC)G5 (1:1,000; bs-5013R; BIOSS, Beijing, China), ABCG8 (1:1,000; bs-10149R; BIOSS), farnesoid-X receptor (FXR; 1:1,000; NR1H4, YN2161; ImmunoWay Biotechnology Company, Plano, TX, USA) cholesterol $7 \alpha$-hydroxylase 1 (CYP7A1; 1:1,000; sc-14426, Santa Cruz Biotechnology, Inc.), and GAPDH (1:1,000; B661204-0001, Sangon Biotech Co., Ltd., Shanghai, China); the membranes were washed three times with TBST (10 min each time) and subsequently incubated with HRP-conjugated rabbit anti-mouse immunoglobulin G secondary antibodies (cat. no. 58802; 1:1,000; Cell Signaling Technology, Inc., Danvers, MA, USA) for $1 \mathrm{~h}$ at room temperature. Visualization was performed with an enhanced chemiluminescence detection reagent (GE Healthcare, Chicago, IL, USA), and GAPDH was used as an internal control. Expression levels were quantified using Image J 1.46r image analysis software (National Institutes of Health, Bethesda, MD, USA).

Statistical analysis. Data are presented as the mean \pm standard error of the mean. One-way analysis of variance with Fisher's least-significant difference post hoc analysis for multiple comparisons was applied to compare differences among multiple groups. $\mathrm{P}<0.05$ was considered to indicate a statistically significant difference.

\section{Results}

Effects of ML on serum lipid levels and fecal TC and TBA levels. As presented in Fig. 1, serum TC $(\mathrm{P}<0.01), \mathrm{TG}$
$(\mathrm{P}<0.05)$ and LDL-C $(\mathrm{P}<0.01)$ levels, and fecal TC $(\mathrm{P}<0.05)$ and TBA $(\mathrm{P}<0.01)$ levels in the model group were significantly increased, compared with the normal group, whereas serum HDL-C levels were significantly decreased $(\mathrm{P}<0.01)$. Compared with the model group, atorvastatin significantly lowered serum TC and LDL-C levels $(\mathrm{P}<0.01)$, however no other significant differences were observed. ML treatment reduced TC (0.6 and $0.3 \mathrm{~g} / \mathrm{kg} ; \mathrm{P}<0.05)$, TG $(0.6 \mathrm{~g} / \mathrm{kg}, \mathrm{P}<0.01$; $0.3 \mathrm{~g} / \mathrm{kg}, \mathrm{P}<0.05)$ and LDL-C (all, $\mathrm{P}<0.01)$ concentrations, and $0.6 \mathrm{~g} / \mathrm{kg}$ ML significantly increased HDL-C levels $(\mathrm{P}<0.05)$. Conversely, in ML treatment groups, fecal TC $(0.6 \mathrm{~g} / \mathrm{kg}$, $\mathrm{P}<0.01 ; 0.3 \mathrm{~g} / \mathrm{kg}, \mathrm{P}<0.05)$ and TBA $(0.6$ and $0.3 \mathrm{~g} / \mathrm{kg}, \mathrm{P}<0.01)$ levels were significantly increased, compared with the model group. These findings suggest that the serum cholesterol reduction of ML may be associated with the excretion of TC and TBA.

Effects of ML on liver pathological changes and hepatic lipidosis. Serum AST activity was significantly increased in model rats compared with the normal group $(\mathrm{P}<0.01 ;$ Fig. $2 \mathrm{~A})$, and ALT activity exhibited a similar, marked trend (Fig. 2B). Representative images of liver histology demonstrated that normal rats presented normal liver histology, as hepatocytes were observed with a common radial array encircling the central veins and no hepatocyte lipid degeneration was observed (Fig. 2C-a). In model rats, the lobular structures of hepatocytes were disrupted and inflammatory cell infiltration was observed (Fig. 2C-b). Atorvastatin significantly reduced serum AST and ALT activity compared with the model group (Fig. 2A and B), and notably improved hepatocyte lipid degeneration (Fig. 2C-c). ML reduced serum AST activity in a dose-dependent manner $(0.6 \mathrm{~g} / \mathrm{kg}, \mathrm{P}<0.05 ; 0.3 \mathrm{~g} / \mathrm{kg}, \mathrm{P}<0.01$; Fig. 2A), and induced a marked decrease in ALT activity (Fig. 2B), compared with the model group. ML also markedly reduced neutrophil infiltration (Fig. 2C-d-f).

Liver TC (Fig. 3A) and TBA (Fig. 3B) levels in the model group were significantly increased $(\mathrm{P}<0.01$ and $<0.05$, respectively) compared with normal rats, and liver TG exhibited a similar, marked increase (Fig. 3A). Lipid droplets were visible in the hepatic plates, and oil red $\mathrm{O}$ staining revealed a pale pink staining in the normal rat hepatic tissue (Fig. 3C-a), while the model rats exhibited severe hepatic steatosis (Fig. 3C-b). ML significantly decreased liver TC (0.9 and $0.6 \mathrm{~g} / \mathrm{kg}, \mathrm{P}<0.01 ; 0.3 \mathrm{~g} / \mathrm{kg}, \mathrm{P}<0.05$; Fig. 3A) and TBA $(0.9 \mathrm{~g} / \mathrm{kg}, \mathrm{P}<0.05$; Fig. 3B) levels, and the color and density of oil red $\mathrm{O}$ staining of the liver was weakened, indicating that Atorastatin and ML markedly alleviated hepatocyte lipid degeneration (Fig. 3C-c-f).

Effects of ML on expressions of cholesterol absorption-related proteins in liver. According to the effects of ML on serum and liver lipid level and fecal TC and TBA level, two of the ML treated groups ( 0.6 and $0.3 \mathrm{~g} / \mathrm{kg}$ ) were chosen to observe the expression of cholesterol absorption and excretion-related proteins in the liver.

LDL-R and SR-BI are associated with the transportation of cholesterol from peripheral blood to the liver $(26,27)$. As demonstrated in representative images of immunohistochemistry, LDL-R protein was predominantly located at the hepatic portal vein area (Fig. 4A). In the normal, Atorastatin and ML groups, 

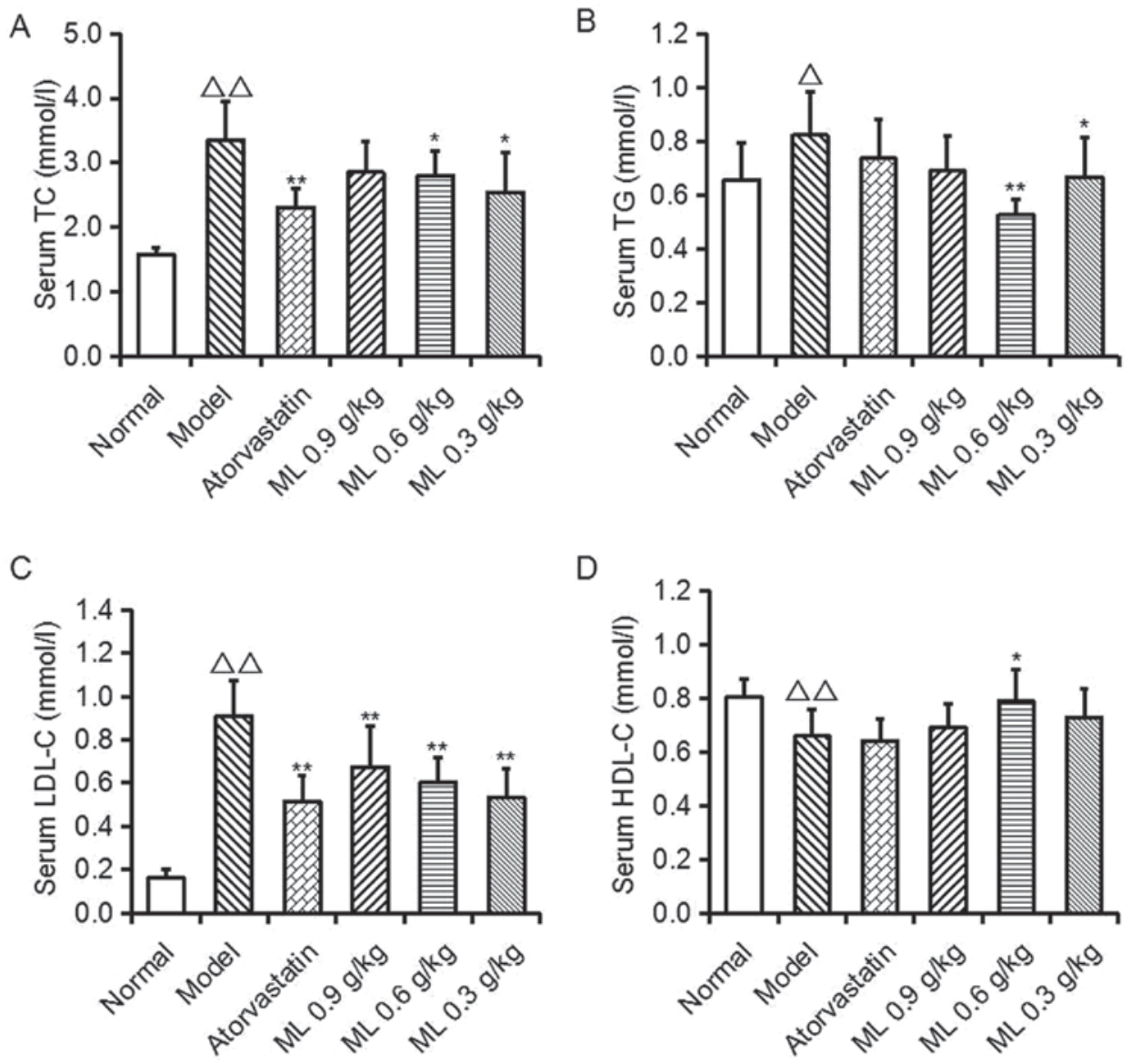

D
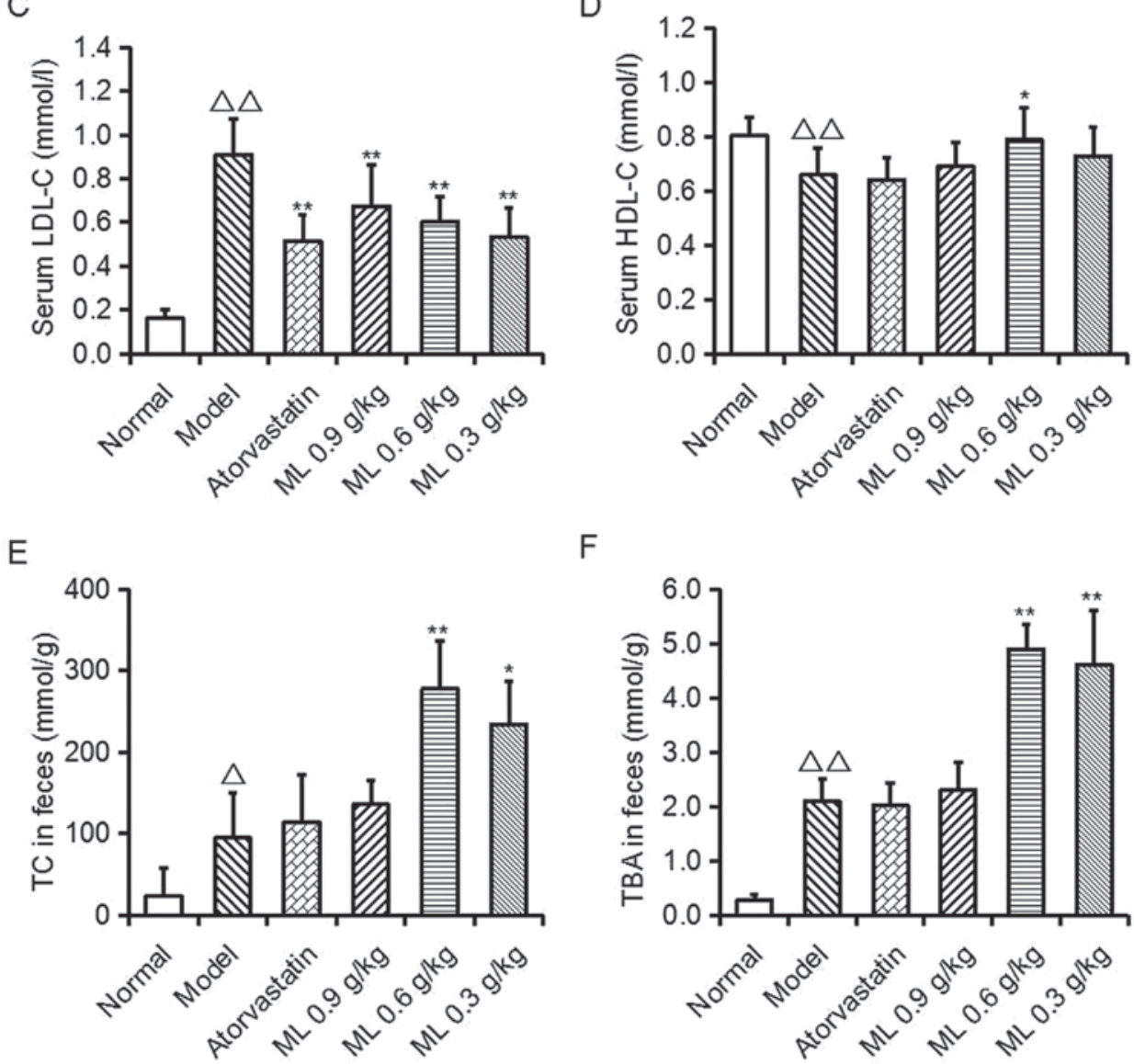

$\mathrm{F}$

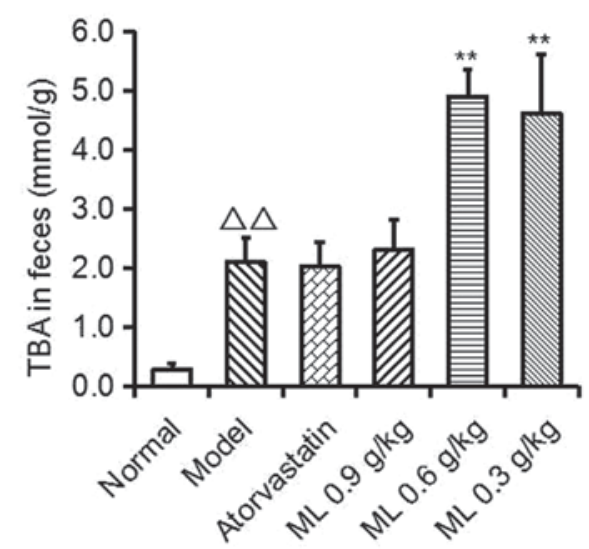

Figure 1. Effects of ML on serum lipid levels and fecal TC and TBA levels in high-fat diet-fed rats. (A) Serum TC levels; (B) serum TG levels; (C) serum LDL-C levels; (D) serum HDL-C levels; (E) fecal TC levels; (F) fecal TBA levels. Data are presented as the mean \pm standard error of the mean ( $\mathrm{n}=8$ ). ${ }^{\wedge} \mathrm{P}<0.05$, ${ }^{\Delta} \mathrm{P}<0.01$, vs. normal; ${ }^{*} \mathrm{P}<0.05,{ }^{* *} \mathrm{P}<0.01$ vs. model. ML, mulberry leaf; TC, total cholesterol; TBA, total bile acid; TG, triglyceride; LDL-C, low-density lipoprotein cholesterol; HDL-C, high-density lipoprotein cholesterol.

LDL-R was primarily located at the hepatocytes close to the vein (Fig. 4A-a, c and d) while in the model group it was observed in a wider range (Fig. 4A-b). SR-BI was predominantly located at the hepatocyte membrane and no notable differences were observed between the groups (Fig. 4B-a-d). Western blotting revealed that the SR-BI protein expression in model rats was significantly decreased compared with normal rats $(\mathrm{P}<0.01)$, whereas LDL-R expression was significantly increased $(\mathrm{P}<0.01$; Fig. 5A and B). Atorastatin and ML significantly reduced SR-BI $(0.6 \mathrm{~g} / \mathrm{kg}, \mathrm{P}<0.01)$ and LDL-R (both, $\mathrm{P}<0.01)$ levels compared with the model group (Fig. 5A and B).
Effects of ML on expressions of cholesterol excretion related proteins in liver. ABCG5, ABCG8, FXR, PPAR $\alpha$ and CYP7A1 are associated with the conversion of cholesterol into TBA in the liver, and the excretion of TBA in feces. Compared with the normal group, a decrease was observed in ABCG5 and ABCG8 $(\mathrm{P}<0.01)$ protein expression in the model group (Fig. 5A and $\mathrm{C}$ ), and the ratio of ABCG5/ABCG8 in the model group was significantly increased, compared with that of the normal group ( $\mathrm{P}<0.01$; Fig. 5D). This indicated that the balance of ABCG5 and ABCG8 was changed. ABCG5 and ABCG8 protein expressions were significantly decreased in Atorastatin and ML-treated 
A
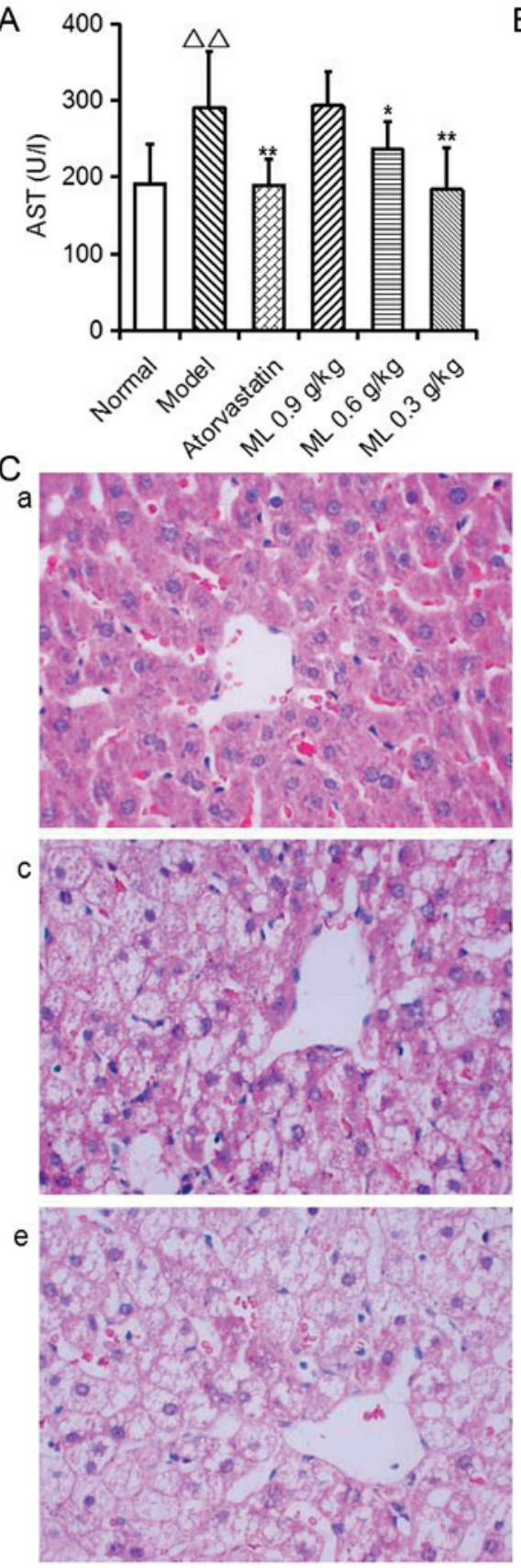
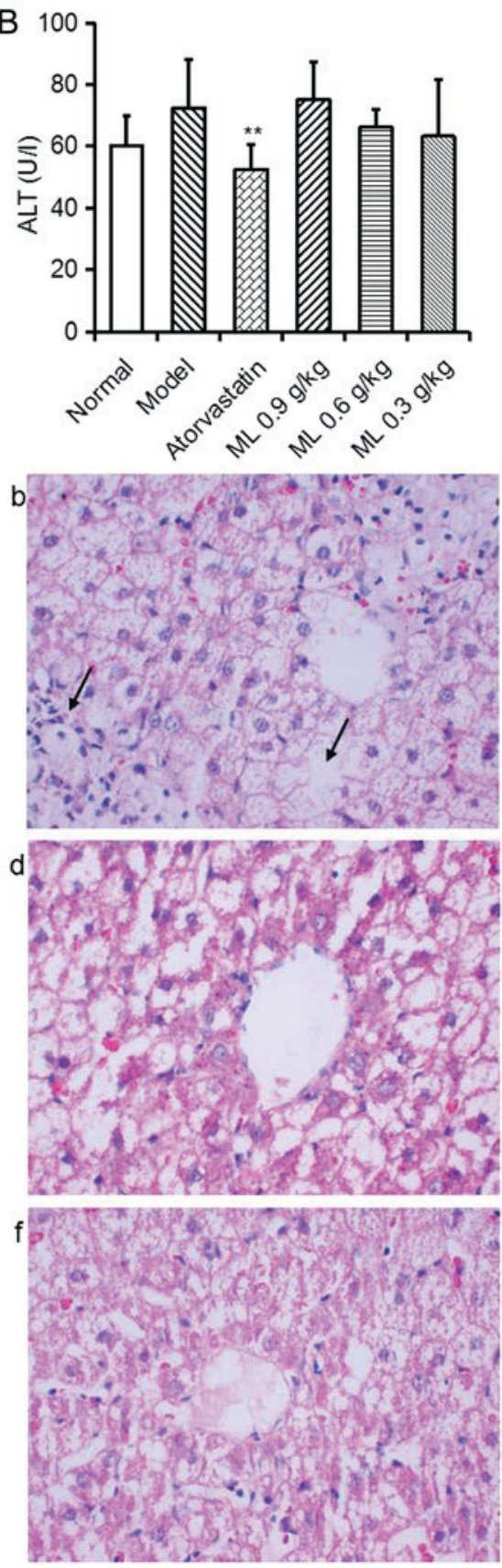

Figure 2. Effects of ML on liver functions in high-fat diet-fed rats. (A) Serum AST activities; (B) serum ALT activities. (C) Representative images of liver histology. The black arrow indicates neutrophil infiltration or hepatocyte structure injury (hematoxylin-eosin staining; magnification, $\mathrm{x} 200$ ). (a) Normal; (b) model; (c) atorvastatin; (d) $0.9 \mathrm{~g} / \mathrm{kg} \mathrm{ML;} \mathrm{(e)} 0.6 \mathrm{~g} / \mathrm{kg} \mathrm{ML}$; (f) $0.3 \mathrm{~g} / \mathrm{kg}$ ML. Data are presented as the mean \pm standard error of the mean (n=8). ${ }^{\Delta \Delta} \mathrm{P}<0.01$, vs. normal; ${ }^{*} \mathrm{P}<0.05,{ }^{* *} \mathrm{P}<0.01$ vs. model. ML, mulberry leaf; AST, aspartate aminotransferase; ALT, alanine aminotransferase.

rats compared with the model group (both $0.6 \mathrm{~g} / \mathrm{kg}, \mathrm{P}<0.05$; $0.3 \mathrm{~g} / \mathrm{kg}, \mathrm{P}<0.01)$, however the ratio of ABCG5/ABCG8 was significantly lower compared with that of the model rats (both, $\mathrm{P}<0.01$ ), indicating that ML was able to maintain the balance of ABCG5 and ABCG8 expression in the liver.

Compared with normal rats, expression of PPAR $\alpha$ protein was increased significantly in the model group $(\mathrm{P}<0.05)$, whereas FXR and CYP7A1 were significantly decreased (both, $\mathrm{P}<0.01$; Fig. 5E). ML at 0.6 and $0.3 \mathrm{~g} / \mathrm{kg}$ significantly decreased the expression of PPAR $\alpha$ and FXR protein in liver, compared with model rats (all, $\mathrm{P}<0.01$ ), whereas $0.3 \mathrm{~g} / \mathrm{kg} \mathrm{ML}$ significantly increased the protein expression of CYP7A1 $(\mathrm{P}<0.01$; Fig. 5A and $\mathrm{E})$.

\section{Discussion}

Hypercholesterolemia accompanied by high serum LDL-C and low HDL-C is a main factor for the development of atherosclerotic disease $(28,29)$. Excess diet-derived cholesterol is the primary cause for hypercholesterolemia (30). In the present study, rats fed with HFD for 5 weeks exhibited significantly increased serum TC and LDL-C levels. ML treatment significantly reduced the accumulation of TC in plasma and the liver, and alleviated hepatocyte lipid deposition in high-fat diet-fed rats. $\mathrm{H} \& \mathrm{E}$ and oil red $\mathrm{O}$ staining images presented lipid accumulation in the livers of rats fed an HFD, and a marked increase of liver TG levels was 
A

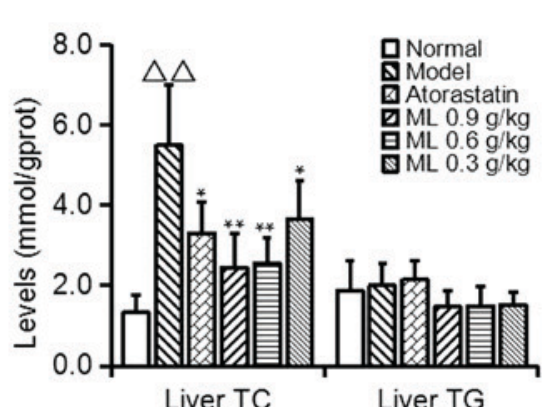

C

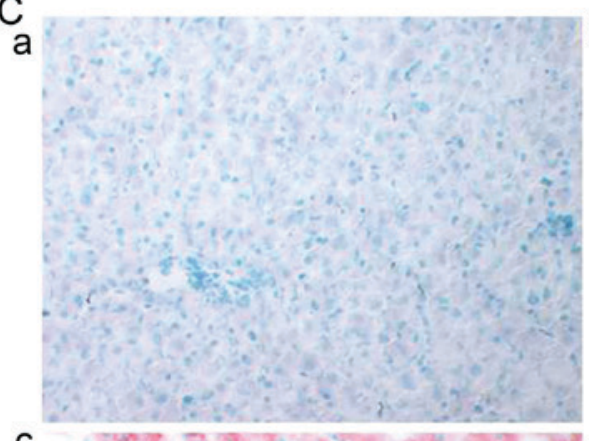

C

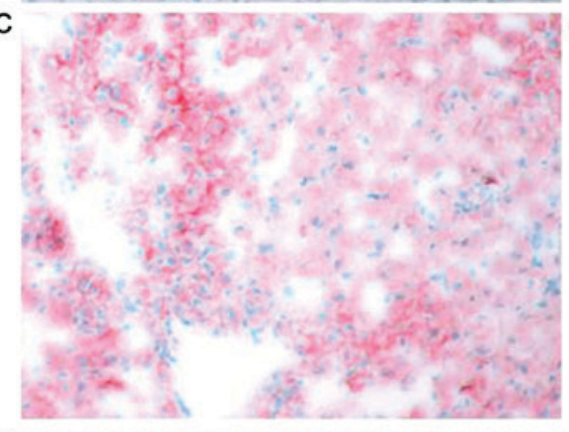

e

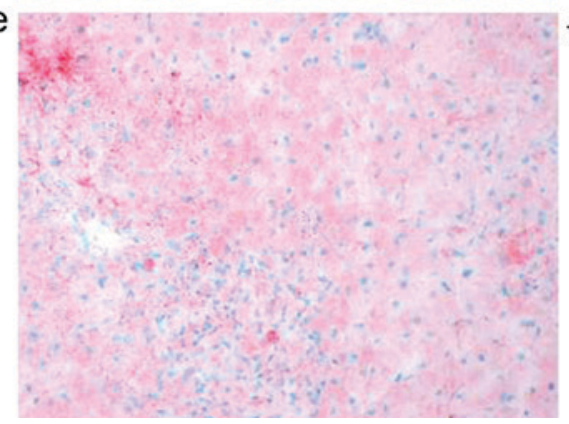

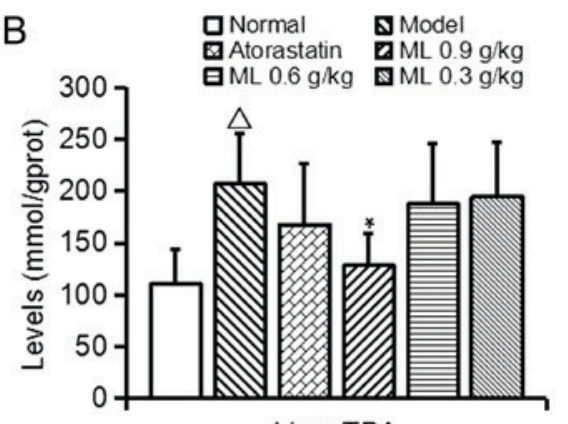

Liver TBA
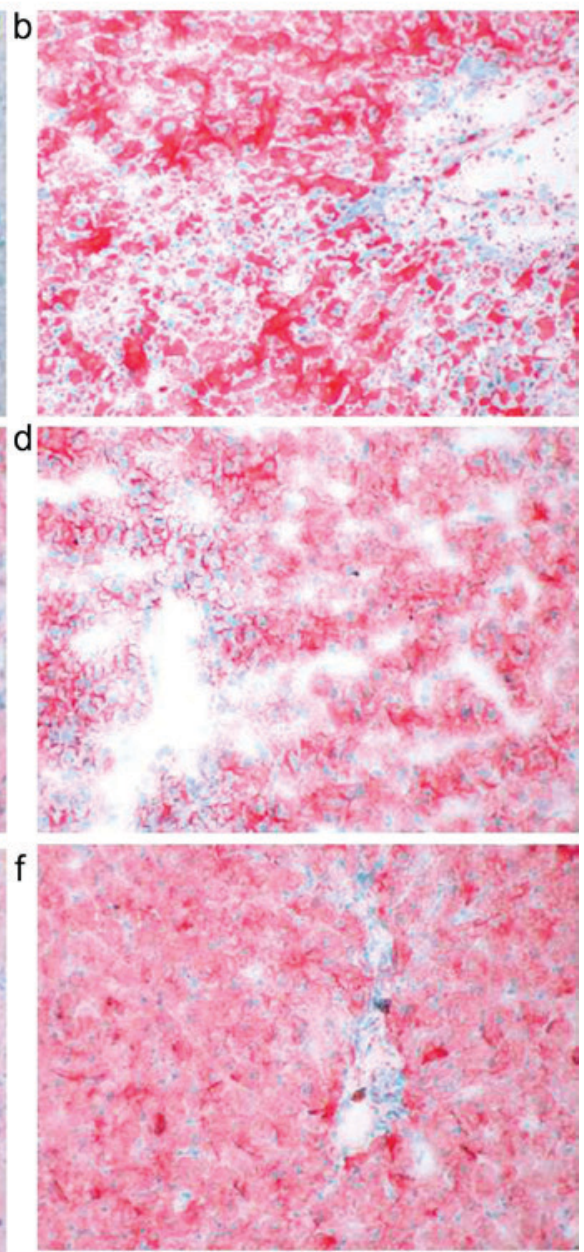

Figure 3. Effects of ML on liver fatty degeneration in high-fat diet-fed rats. (A) Liver TC and TG levels; (B) liver TBA levels. (C) Representative images of hepatocyte lipid droplet (oil red O staining; original magnification, x200) (a) Normal; (b) model; (c) atorvastatin; (d) 0.9 g/kg ML; (e) $0.6 \mathrm{~g} / \mathrm{kg} \mathrm{ML;} \mathrm{(f)} 0.3 \mathrm{~g} / \mathrm{kg}$ ML. Data are presented as the mean \pm standard error of the mean $(\mathrm{n}=8)$. ${ }^{\Delta} \mathrm{P}<0.05,{ }^{\Delta \Delta} \mathrm{P}<0.01$, vs. normal; ${ }^{*} \mathrm{P}<0.05$, ${ }^{* *} \mathrm{P}<0.01$ vs. model. ML, mulberry leaf; TC, total cholesterol; TG, triglyceride; TBA, total bile acid.

observed in the HFD group compared with the normal group. There are two potential reasons for these findings: i) Intra-group variance induced a non-significant difference between HFD group and normal group; and ii) HFD, which increased the serum TC level, induced TC accumulation in the liver.

$\mathrm{RCT}$ is a process that encompasses the transport of excess cholesterol from peripheral tissues to the liver for biliary and fecal excretion (31). It is an effective method for cholesterol homeostasis in vivo (23) and a protective mechanism to contract atherosclerotic injury (32). Various cholesterol transporters, including SR-BI, ABCG5, ABCG8 and CYP7A1 are associated with RCT, and increase cholesterol transportation and conversion (33). Therefore, medicines which regulate expression of these proteins are potential candidates for hypercholesterolemia treatment.

LDL-R has a role in regulating plasma cholesterol level and cholesterol homeostasis by limiting hepatic uptake of circulating cholesterol (29). However, contrary to recent findings (32), the present study demonstrated that the expression of LDL-R in liver cells was significantly increased in rats with hyperlipidemia, whereas it was decreased in ML-treated rats. The LDL-R pathway is complex $(34,35)$, and with the previous identification of 

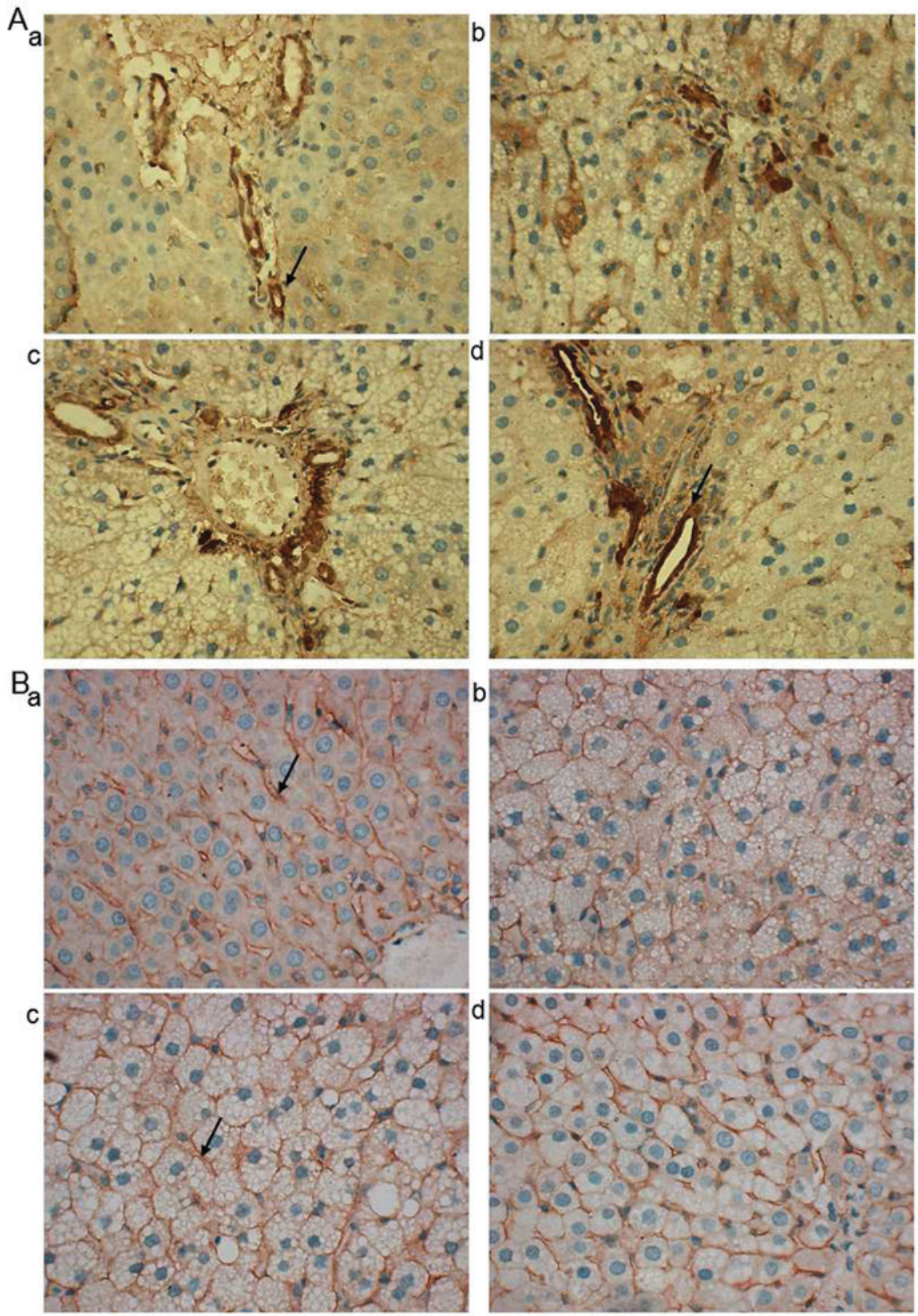

Figure 4. Localization of SR-BI and LDL-R protein expression in livers of high-fat diet-fed rats. (A) Representative immunohistochemistry images of SR-BI in liver (HRP/DAB staining; original magnification, x200). (B) Representative immunohistochemistry images of LDL-R in liver (HRP/DAB staining; original magnification, x200). (a) Normal; (b) model; (c) atorvastatin; (d) ML. SR-BI, scavenger receptor class B type I; LDL-R, low-density lipoprotein receptor; HRP, horseradish peroxidase; DAB, 3,3'-diaminobenzidine.

the LDL-R-proprotein convertase subtilisin/kexin type 9 (PCSK9)-LDL axis in the LDL-R pathway for cholesterol homeostasis, Kosenko et al (36) demonstrated that plasma LDL particles reduce PCSK9-mediated LDL-R degradation in a dose-dependent manner by binding to PCSK9. This finding suggested that decreased plasma LDL may induce a lower expression of LDLR in hepatocytes. However, whether there is an association among serum LDL, hepatic LDL-R, PCSK9 and ML required further study.

SR-BI has a crucial role in cholesterol homeostasis and hepatic SR-BI mediates the final step in RCT via the uptake of HDL-C for routing to the bile $(27,37)$. The suppression of hepatic SR-BI expression impairs HDL-mediated RCT and induces hypercholesterolemia $(38,39)$. In the present study, it was demonstrated that SR-BI protein expression in the liver exhibited a decrease in model rats and ML treatment did not block this decline, which suggested that the SR-BI signal pathway may not be the target of ML in mediating the transportation of cholesterol.

ABCG5 and ABCG8 are expressed in the liver and intestine. They typically form a heterodimer in the endoplasmic reticulum, which pumps neutral sterols back into the gut lumen to prevent the accumulation of other sterols $(40,41)$ and partially contributes to the trans-intestinal cholesterol efflux pathway (42). ABCG5/ABCG8 promotes SR-BI-transported cholesterol excretion in the bile, and eventually in feces, to prohibit the development of hypercholesterolemia, and inactivation of either protein induces sitosterolemia (43). In 
A $\begin{array}{rlllll}\text { HFD } & - & + & + & + & + \\ \text { ATORVASTATIN } & - & - & + & - & - \\ \text { ML } 0.6 \mathrm{~g} / \mathrm{kg} & - & - & - & + & - \\ \mathrm{ML} 0.3 \mathrm{~g} / \mathrm{kg} & - & - & - & - & +\end{array}$

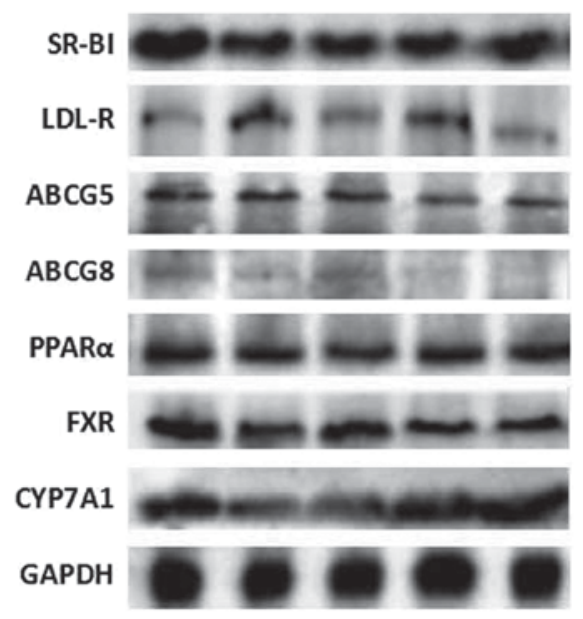

D

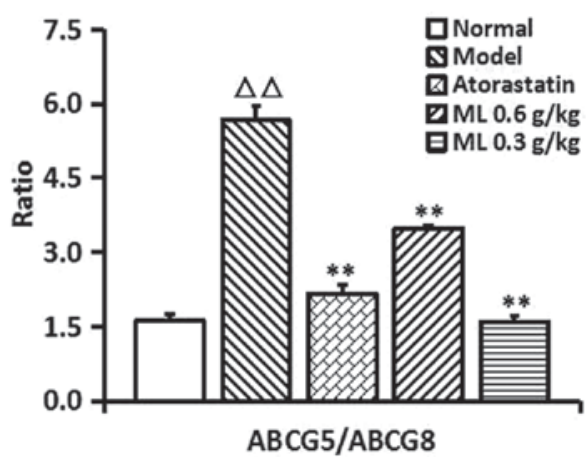

B

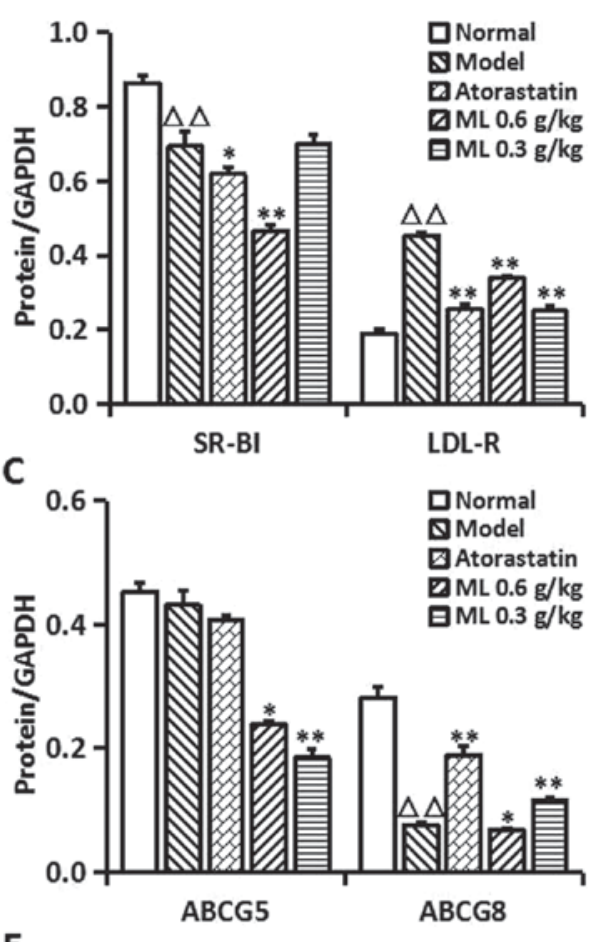

E

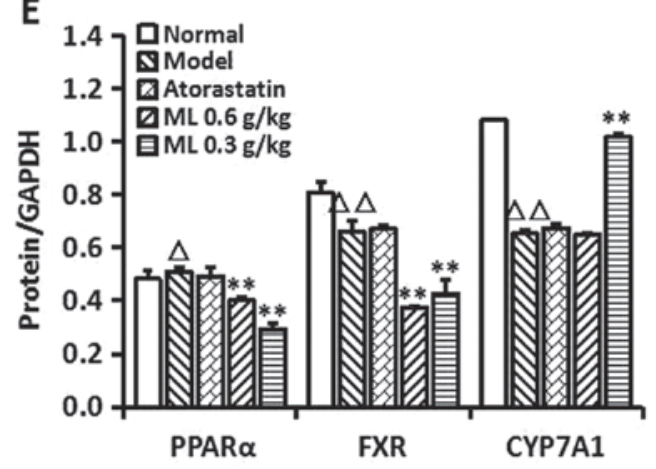

Figure 5. Expressions of cholesterol absorption, conversion and bile acid excretion-associated proteins in the livers of HFD-fed rats. (A) Western blotting images of SR-BI and LDL-R, ABCG5, ABCG8, PPAR $\alpha$, FXR and CYP7A1 protein expression. (B) Relative expression of SR-BI and LDL-R protein. (C) Relative expression of ABCG5 and ABCG8 protein. (D) Ratio of ABCG5/ABDG8 expression. (E) Relative expression of PPAR $\alpha$, FXR and CYP7A1 protein. Data are presented as the mean \pm standard error of the mean $(\mathrm{n}=8) .{ }^{\Delta} \mathrm{P}<0.05,{ }^{\Delta \Delta} \mathrm{P}<0.01$, vs. normal; ${ }^{*} \mathrm{P}<0.05,{ }^{* *} \mathrm{P}<0.01$ vs. model. SR-BI, scavenger receptor class $\mathrm{B}$ type I; LDL-R, low-density lipoprotein receptor; ABCG5, ATP-binding cassette transporter G5; ABCG8, ATP-binding cassette transporter G8; PPAR $\alpha$, peroxisome proliferator-activated receptor- $\alpha$; FXR, farnesoid-X receptor; CYP7A1, cholesterol 7 $\alpha$-hydroxylase 1; HFD, high-fat diet; ML, mulberry leaf.

the current study, TC and TBA levels in the liver and feces were significantly increased in model rats compared with the normal group; in ML-treated rats, liver TC and TBA levels were significantly decreased while fecal TC and TBA levels were significantly increased compared with the model rats, indicating that in addition to the compensatory excretion of cholesterol in rats with HFD intake, ML promoted the clearance of cholesterol by TBA from feces in rats with hypercholesterolemia. Western blotting results demonstrated that ML could maintain the balance of ABCG5 and ABCG8 protein expression in the liver. Together, these findings suggested that maintaining the stabilization and activity of ABCG5/ABCG8 protein may be a potential mechanism for ML to lower serum cholesterol.

The conversion of cholesterol into BA via intestinal and biliary lumen for fecal excretion is the final step to remove cholesterol from the body. CYP7A1 is a rate-limiting enzyme in cholesterol conversion to BA. Promoting CYP7A1 expression accelerates the cholesterol conversion into BA and the excretion in feces $(44,45)$. PPAR $\alpha$ serves a role in the clearance of circulating cholesterol via upregulating CYP7A1 expression in hepatocytes (45). Farnesoid-X receptor (FXR) is an intracellular BA receptor, which can be activated by $\mathrm{BA}$, and has a pivotal role for BA and lipid homeostasis; overexpression of FXR decreases BA pools and reduces fecal BA excretion (46) due to the suppression of hepatic CYP7A1 (47). In the present study, 5-week HFD intake increased PPAR $\alpha$ protein expression, and decreased FXR and CYP7A1 expression in liver, whereas increased levels of TC and TBA in feces were observed. ML treatment markedly inhibited PPAR $\alpha$ and FXR protein expression, upregulated CYP7A1 expression, increased fecal TBA levels, and simultaneously reduced cholesterol in the liver and plasma. Together, these findings suggest that ML may promote the 
conversion of cholesterol into BA and excretion to accelerate the clearance of circulating cholesterol. The present study focused primarily on extrinsic cholesterol excretion from hepatocytes, and the effect of ML on cholesterol synthesis will be considered in future studies. Meanwhile, changes in liver and fecal TBA levels provide rationale that regulating enterohepatic circulation of BA may be a potential mechanism of ML on reduced serum TC levels.

As detailed above, ML exhibited a beneficial effect on anti hyperlipidemia, but no obvious dose-effect association was observed on serum TG and HDL-C levels, and fecal TC and TBA levels. Previous studies have demonstrated that ML contains 1-dexoynokifimycin (48), polyphenols and flavonoids (49), which are active components on blood lipid regulation, anti-atherosclerosis and anti-oxidation (50). In these studies, the test sample was administered an extract of ML in which the active components were enriched to induce a more notable effect. ML is edible and may be used to make tea, which may be a convenient method to prevent hyperlipidemia in the future. In the present study, ML powder was produced via a process of hot air drying and ball milling technology as the test sample. As this sample contained all the components of ML, it was not possible to identify an exact dose-effect relationship, but the present results have identified an effective dosage for intake. However, if ML is to be considered as a candidate medicine for hypercholesterolemia treatment, it is essential that the chemical material basis and the dose-effect relationship are elucidated.

The findings of the present study suggest a positive role of ML on cholesterol clearance by promoting cholesterol and TBA execration via FXR- and CYP7A1-mediated pathways, and that RCT regulation may be a potential mechanism of $\mathrm{ML}$ on anti hypercholesterolemia.

\section{Acknowledgements}

The authors would like to thank Dr Gao Jianli of Zhejiang Chinese Medical University (Hangzhou, China) for their assistance and revision of the manuscript.

\section{Funding}

The present study was supported by Zhejiang Provincial Natural Science Foundation of China (grant no. LY15H280007), National Natural Science Foundation of China (grant no. 81503328), and The Zhejiang Provincial Key Laboratory Project (grant no. 2012E10002).

\section{Availability of data and materials}

The datasets used and analyzed during the current study are available from the corresponding author on reasonable request.

\section{Authors' contributions}

JH designed and organized the study. YW and LL performed the animal experiments. CY performed the western blot analysis. ZL performed the data analysis and wrote the manuscript.

\section{Ethics approval and consent to participate}

The present study was approved by the Ethics Committee of Zhejiang Chinese Medical University (Hangzhou, China).

\section{Consent for publication}

Not applicable.

\section{Competing interests}

The authors declare that they have no competing interests.

\section{References}

1. Navar-Boggan AM, Peterson ED, D'Agostino RB Sr, Neely B, Sniderman AD and Pencina MJ: Hyperlipidemia in early adulthood increases long-term risk of coronary heart disease. Circulation 131: 451-458, 2015.

2. Garcia GH, Liu JN, Wong A, Cordasco F, Dines DM, Dines JS, Gulotta LV and Warren R: Hyperlipidemia increases the risk of retear after arthroscopic rotator cuff repair. J Shoulder Elbow Surg 26: 2086-2090, 2017.

3. Bjornsson E, Jacobsen EI and Kalaitzakis E: Hepatotoxicity associated with statins: Reports of idiosyncratic liver injury post-marketing. J Hepatol 56: 374-380, 2012.

4. Graham DJ, Staffa JA, Shatin D, Andrade SE, Schech SD, La Grenade L, Gurwitz JH, Chan KA, Goodman MJ and Platt R: Incidence of hospitalized rhabdomyolysis in patients treated with lipid-lowering drugs. JAMA 292: 2585-2590, 2004.

5. Sakamoto K, Honda T, Yokoya S, Waguri S and Kimura J: Rab-small GTPases are involved in fluvastatin and pravastatin-induced vacuolation in rat skeletal myofibers. FASEB J 21: 4087-4094, 2007

6. Chu SM, Shih WT, Yang YH, Chen PC and Chu YH: Use of traditional Chinese medicine in patients with hyperlipidemia: A population-based study in Taiwan. J Ethnopharmacol 168: 129-135, 2015

7. Cai S, Sun W, Fan Y, Guo X, Xu G, Xu T, Hou Y, Zhao B, Feng X and Liu T: Effect of mulberry leaf (Folium Mori) on insulin resistance via IRS-1/PI3K/Glut-4 signalling pathway in type 2 diabetes mellitus rats. Pharm Biol 54: 2685-2691, 2016.

8. Salemi Z, Barzin Tond S, Fallah S, Shojaii A and Seifi M: The effect of Morus alba leaves extract and powder on resistin levels and liver transaminase enzymes activities in diabetes. Cell Mol Biol (Noisy-le-Grand) 62: 112-118, 2016.

9. Zhang Y, Ren C, Lu G, Cui W, Mu Z, Gao H and Wang Y: Purification, characterization and anti-diabetic activity of a polysaccharide from mulberry leaf. Regul Toxicol Pharmacol 70: 687-695, 2014.

10. Khan MA, Rahman AA, Islam S, Khandokhar P, Parvin S, Islam MB, Hossain M, Rashid M, Sadik G, Nasrin S, et al: A comparative study on the antioxidant activity of methanolic extracts from different parts of Morus alba L. (Moraceae). BMC Res Notes 6: 24, 2013.

11. Lee YJ, Hsu JD, Lin WL, Kao SH and Wang CJ: Upregulation of caveolin-1 by mulberry leaf extract and its major components, chlorogenic acid derivatives, attenuates alcoholic steatohepatitis via inhibition of oxidative stress. Food Funct 8: 397-405, 2017.

12. Jeong JW, Lee HH, Lee KW, Kim KY, Kim SG, Hong SH, Kim GY, Park C, Kim HK, Choi YW and Choi YH: Mori folium inhibits interleukin-1 $\beta$-induced expression of matrix metalloproteinases and inflammatory mediators by suppressing the activation of NF- $\kappa$ B and p38 MAPK in SW1353 human chondrocytes. Int J Mol Med 37: 452-460, 2016.

13. Ann JY, Eo H and Lim Y: Mulberry leaves (Morus alba L.) ameliorate obesity-induced hepatic lipogenesis, fibrosis, and oxidative stress in high-fat diet-fed mice. Genes Nutr 10: 46, 2015.

14. Sugimoto M, Arai H, Tamura Y, Murayama T, Khaengkhan $P$, Nishio T, Ono K, Ariyasu H, Akamizu T, Ueda Y, et al: Mulberry leaf ameliorates the expression profile of adipocytokines by inhibiting oxidative stress in white adipose tissue in $\mathrm{db} / \mathrm{db}$ mice. Atherosclerosis 204: 388-394, 2009. 
15. Enkhmaa B, Shiwaku K, Katsube T, Kitajima K, Anuurad E, Yamasaki M and Yamane Y: Mulberry (Morus alba L.) leaves and their major flavonol quercetin 3-(6-malonylglucoside) attenuate atherosclerotic lesion development in LDL receptor-deficient mice. J Nutr 135: 729-734, 2005.

16. Aramwit P, Petcharat K and Supasyndh O: Efficacy of mulberry leaf tablets in patients with mild dyslipidemia. Phytother Res 25 365-369, 2011.

17. Aramwit P, Supasyndh O, Siritienthong $T$ and Bang N: Mulberry leaf reduces oxidation and C-reactive protein level in patients with mild dyslipidemia. Biomed Res Int 2013: 787981, 2013.

18. Kobayashi Y, Miyazawa M, Kamei A, Abe K and Kojima T: Ameliorative effects of mulberry (Morus alba L.) leaves on hyperlipidemia in rats fed a high-fat diet: Induction of fatty acid oxidation, inhibition of lipogenesis, and suppression of oxidative stress. Biosci Biotechnol Biochem 74: 2385-2395, 2010.

19. Lou Z, Zhang G, Su J, Xia B, Yu J and Yan M: Effect of green tea and mulberry leaf powder on serum lipid profile in rats with hyperlipidemia. Zhongchengyao 38: 1594-1597, 2016 (in Chinese).

20. Trimarco V, Izzo R, Stabile E, Rozza F, Santoro M, Manzi MV, Serino F, Schiattarella GG, Esposito G and Trimarco B: Effects of a new combination of nutraceuticals with Morus alba on lipid profile, insulin sensitivity and endotelial function in dyslipidemic subjects. A cross-over, randomized, double-blind trial. High Blood Press Cardiovasc Prev 22: 149-154, 2015.

21. Valacchi G, Belmonte G, Miracco C, Eo H and Lim Y: Effect of combined mulberry leaf and fruit extract on liver and skin cholesterol transporters in high fat diet-induced obese mice. Nutr Res Pract 8: 20-26, 2014.

22. Riwanto $M$ and Landmesser U: High density lipoproteins and endothelial functions: Mechanistic insights and alterations in cardiovascular disease. J Lipid Res 54: 3227-3243, 2013.

23. van der Velde AE, Vrins CL, van den Oever K, Seemann I, Oude Elferink RP, van Eck M, Kuipers F and Groen AK: Regulation of direct transintestinal cholesterol excretion in mice. Am J Physiol Gastrointest Liver Physiol 295: G203-G208, 2008.

24. The State Science and Technology Commission of China, Regulations on the management of laboratory animals. Shiyong Qiguan Yizhi Zazhi 4: 66-67,2016 (in Chinese).

25. Ministry of Science and Technology of the People's Republic of China. (2018). Methods for managing experimental animal licenses (trial). [online] Available at: http://www.most.gov.cn/ fggw/zfwj/zfwj2001/zf01yw/zf01kjjh/200312/t20031209_31332. htm [Accessed July 30, 2015 ]

26. Brown MS and Goldstein JL: A receptor-mediated pathway for cholesterol homeostasis. Science 232: 34-47, 1986.

27. Huby T, Doucet C, Dachet C, Ouzilleau B, Ueda Y, Afzal V, Rubin E, Chapman MJ and Lesnik P: Knockdown expression and hepatic deficiency reveal an atheroprotective role for SR-BI in liver and peripheral tissues. J Clin Invest 116: 2767-2776, 2006.

28. Braamskamp MJ, Hutten BA and Wiegman A: Early initiation of statin treatment in children with familial hypercholesterolaemia. Curr Opin Lipidol 26: 236-239, 2015.

29. Yakushiji E, Ayaori M, Nishida T, Shiotani K, Takiguchi S, Nakaya K, Uto-Kondo H, Ogura M, Sasaki M, Yogo M, et al: Probucol-oxidized products, spiroquinone and diphenoquinone, promote reverse cholesterol transport in mice. Arterioscler Thromb Vasc Biol 36: 591-597, 2016.

30. Wang DQ: Regulation of intestinal cholesterol absorption. Annu Rev Physiol 69: 221-248, 2007

31. Orsoni A, Villard EF, Bruckert E, Robillard P, Carrie A, Bonnefont-Rousselot D, Chapman MJ, Dallinga-Thie GM, Le Goff W and Guerin M: Impact of LDL apheresis on atheroprotective reverse cholesterol transport pathway in familial hypercholesterolemia. J Lipid Res 53: 767-775, 2012.

32. Zhang Y, Si Y, Zhai L, Guo S, Zhao J, Sang H, Pang X, Zhang X, Chen A and Qin S: Celastrus orbiculatus thunb. Reduces lipid accumulation by promoting reverse cholesterol transport in hyperlipidemic mice. Lipids 51: 677-692, 2016.

33. Zhu RG, Sun YD, Hou YT, Fan JG, Chen G and Li TP: Pectin penta-oligogalacturonide reduces cholesterol accumulation by promoting bile acid biosynthesis and excretion in high-cholesterol-fed mice. Chem Biol Interact 272: 153-159, 2017.
34. Maxwell KN, Fisher EA and Breslow JL: Overexpression of PCSK9 accelerates the degradation of the LDLR in a post-endoplasmic reticulum compartment. Proc Natl Acad Sci USA 102: 2069-2074, 2005.

35. Zelcer N, Hong C, Boyadjian R and Tontonoz P: LXR regulates cholesterol uptake through Idol-dependent ubiquitination of the LDL receptor. Science 325: 100-104, 2009.

36. Kosenko T, Golder M, Leblond G, Weng W and Lagace TA: Low density lipoprotein binds to proprotein convertase subtilisin/kexin type-9 (PCSK9) in human plasma and inhibits PCSK9-mediated low density lipoprotein receptor degradation. J Biol Chem 288: 8279-8288, 2013.

37. Ji Y, Wang N, Ramakrishnan R, Sehayek E, Huszar D, Breslow JL and Tall AR: Hepatic scavenger receptor BI promotes rapid clearance of high density lipoprotein free cholesterol and its transport into bile. J Biol Chem 274: 33398-33402, 1999.

38. Braun A, Zhang S, Miettinen HE, Ebrahim S, Holm TM, Vasile E, Post MJ, Yoerger DM, Picard MH, Krieger JL, et al: Probucol prevents early coronary heart disease and death in the high-density lipoprotein receptor SR-BI/apolipoprotein E double knockout mouse. Proc Natl Acad Sci USA 100: 7283-7288, 2003.

39. Chulsky S, Paland N, Lazarovich A and Fuhrman B: Urokinase-type plasminogen activator (uPA) decreases hepatic SR-BI expression and impairs HDL-mediated reverse cholesterol transport. Atherosclerosis 233: 11-18, 2014.

40. Berge KE, Tian H, Graf GA, Yu L, Grishin NV, Schultz J, Kwiterovich P, Shan B, Barnes R and Hobbs HH: Accumulation of dietary cholesterol in sitosterolemia caused by mutations in adjacent ABC transporters. Science 290: 1771-1775, 2000.

41. Wang J, Mitsche MA, Lutjohann D, Cohen JC, Xie XS and Hobbs HH: Relative roles of ABCG5/ABCG8 in liver and intestine. J Lipid Res 56: 319-330, 2015.

42. van der Veen JN, van Dijk TH, Vrins CL, van Meer H, Havinga R, Bijsterveld K, Tietge UJ, Groen AK and Kuipers F: Activation of the liver $\mathrm{X}$ receptor stimulates trans-intestinal excretion of plasma cholesterol. J Biol Chem 284: 19211-19219, 2009.

43. Dikkers A, Freak de Boer J, Annema W, Groen AK and Tietge UJ: Scavenger receptor BI and ABCG5/G8 differentially impact biliary sterol secretion and reverse cholesterol transport in mice. Hepatology 58: 293-303, 2013.

44. Cao Y, Bei W, Hu Y, Cao L, Huang L, Wang L, Luo D, Chen Y, Yao X, He W, et al: Hypocholesterolemia of Rhizoma Coptidis alkaloids is related to the bile acid by up-regulated CYP7A1 in hyperlipidemic rats. Phytomedicine 19: 686-692, 2012.

45. Li T, Matozel M, Boehme S, Kong B, Nilsson LM, Guo G, Ellis E and Chiang JY: Overexpression of cholesterol 7alpha-hydroxylase promotes hepatic bile acid synthesis and secretion and maintains cholesterol homeostasis. Hepatology 53: 996-1006, 2011.

46. Sinal CJ, Tohkin M, Miyata M, Ward JM, Lambert G and Gonzalez FJ: Targeted disruption of the nuclear receptor FXR/BAR impairs bile acid and lipid homeostasis. Cell 102: 731-744, 2000.

47. Cai SY, He H, Nguyen T, Mennone A and Boyer JL: Retinoic acid represses CYP7A1 expression in human hepatocytes and HepG2 cells by FXR/RXR-dependent and independent mechanisms. J Lipid Res 51: 2265-2274, 2010.

48. Kojima Y, Kimura T, Nakagawa K, Asai A, Hasumi K, Oikawa S and Miyazawa T: Effects of mulberry leaf extract rich in 1-deoxynojirimycin on blood lipid profiles in humans. J Clin Biochem Nutr 47: 155-161, 2010.

49. Chan KC, Yang MY, Lin MC, Lee YJ, Chang WC and Wang CJ: Mulberry leaf extract inhibits the development of atherosclerosis in cholesterol-fed rabbits and in cultured aortic vascular smooth muscle cells. J Agric Food Chem 61: 2780-2788, 2013.

50. He X, Fang J, Ruan Y, Wang X, Sun Y, Wu N, Zhao Z, Chang Y, Ning N, Guo H and Huang L: Structures, bioactivities and future prospective of polysaccharides from Morus alba (white mulberry): A review. Food Chem 245: 899-910, 2018.

This work is licensed under a Creative Commons Attribution-NonCommercial-NoDerivatives 4.0 International (CC BY-NC-ND 4.0) License. 